\title{
Efficacy and tolerability of various bowel preparations in diabetic patients: a randomized controlled trial
}

다)(1) $(9$

\author{
Authors \\ Tierney ${ }^{1,2}$ \\ Institutions \\ 1 Veterans Affairs Medical Center, Oklahoma City, \\ Oklahoma, United States \\ 2 Division of Digestive Diseases and Nutrition, \\ Department of Internal Medicine, University of \\ Oklahoma Health Sciences Center, Oklahoma City, \\ Oklahoma, United States
}

Mohammad F. Madhoun ${ }^{1,2}$, Khadija K. Chaudrey ${ }^{1,2}$, Sian S. Chisholm ${ }^{1,2}$, Aftab Ahmed ${ }^{1,2}$, Belinda Frost ${ }^{1}$, William M.

submitted 25.8.2017

accepted after revision 8.2 .2018

\author{
Bibliography \\ DOI https://doi.org/10.1055/a-0650-3908 | \\ Endoscopy International Open 2018; 06: E1157-E1163 \\ (c) Georg Thieme Verlag KG Stuttgart · New York \\ ISSN 2364-3722
}

\section{Corresponding author}

Mohammad F. Madhoun, MD MS, Director of Endoscopy, Veterans Affairs Medical Center, Associate Professor of Medicine, University of Oklahoma Health Sciences Center, Division of Digestive Diseases and Nutrition, Andrews Academic Tower, Suite 7400, 800 Stanton L. Young Blvd, Oklahoma City, OK 73104

Fax: +1-405-271-5803

mohammad-madhoun@ouhsc.edu

\section{ABSTRACT}

Background and study aims Diabetes mellitus (DM) is an independent risk factor for poor bowel preparation prior to colonoscopy. Bisacodyl is a stimulant laxative that may mitigate colonic dysmotility associated with diabetes. We hypothesized that adding bisacodyl to split-dose bowel prep- aration (SDBP) would improve the quality of bowel preparation among patients with diabetes.

Patients and methods Adult outpatients aged 18 to 80 years undergoing colonoscopy were recruited. One hundred and eighty-six patients with diabetes were randomly assigned to 1 of 3 treatment arms: 1) conventional $4 \mathrm{~L}$ of polyethylene glycol electrolyte lavage solution (PEG-ELS; conventional bowel preparation [CBP]); 2) split-dose of $4 \mathrm{~L}$ PEG-ELS (split-dose bowel preparation [SDBP]); or 3) splitdose of $4 \mathrm{~L}$ PEG-ELS preceded by $10 \mathrm{mg}$ of oral bisacodyl 10 (SDBP-B). The primary outcome measure was bowel cleansing as indicated by Boston Bowel Preparation Scale (BBPS) score. Endoscopists were blinded to the preparation used. Secondary outcome measures were safety and patient tolerability.

Results Of the 212 patients randomized, only 186 received assigned bowel preparation. There were no differences among the three study groups with regard to age, indication, duration of DM, insulin use, narcotic use, or presence of end-organ diabetic complications. There was a trend toward better bowel preparation quality among those receiving SDBP and SDBP-B compared to those receiving CBP, but the trend was not statistically significant $\geq 6$ BBPS; $67 \%$ vs. $83 \%$ vs. $75 \%, P=0.1$ ). In terms of safety and tolerability, there were no differences among the three groups.

Conclusion Adding bisacodyl to SDBP does not improve the quality of bowel preparation in patients with DM. Further efforts are needed to optimize colonoscopy bowel preparation in this population.
Clinical.Trials.gov
NCT01533688
TRIAL REGISTRATION: Interventional (Clinical Trial)
NCT01533688 at clinicaltrials.gov

\section{Introduction}

Colonoscopy is an important diagnostic and therapeutic procedure and plays a critical role in diagnosing colonic disorders and screening and prevention of colorectal cancer. The effectiveness of colonoscopy in preventing colorectal cancer depends on detection and removal of adenomatous polyps [1]. Poor bowel preparation and patient intolerance of the procedure are the most important factors contributing to the limitations of colonoscopy [2]. Inadequate bowel cleansing results in incomplete testing, increased potential to miss lesions, increased 
cost, and decreased patient satisfaction [3,4]. One study evaluating colonic preparation quality and detection of neoplasms concluded that bowel preparation is inadequate in almost $25 \%$ of patients undergoing colonoscopy [5]. Diabetes mellitus (DM) is considered an independent risk factor for inadequate bowel preparation in patients receiving conventional or split-dose bowel preparations [6,7]. In the current study, we aimed to evaluate efficacy and tolerability of various bowel preparation regimens in patients with diabetes. We hypothesized that adding bisacodyl to split-dose polyethylene glycol electrolyte lavage solution (PEG-ELS) could improve bowel cleansing and tolerability in patients with diabetes.

\section{Patients and methods}

This was a prospective, randomized, endoscopist-blinded clinical trial conducted at the Oklahoma City Veterans Affairs Medical Center from August 2012 to October 2016. The study was approved by the Institutional Review Board (IRB) of the University of Oklahoma Health Sciences Center and the Research and Development Office at the Veterans Affairs Medical Center. The study was registered at ClinicalTrial.gov (NCT01533688). All authors of this study had access to the study data and had reviewed and approved the final manuscript.

\section{Participants}

Adult diabetic outpatients referred for elective colonoscopy were offered an informational classroom session before undergoing colonoscopy. During the classroom session, patients were offered the opportunity to enroll in the study. If they agreed to participate, informed consent was obtained. Exclusion criteria included: diet-controlled DM, age younger than 18 years, known or suspected pregnancy, known or suspected renal failure, creatinine clearance $<30$, unstable angina, acute coronary syndrome, decompensated congestive heart failure, ascites, known or suspected bowel obstruction, major psychiatric illness, known allergies to PEG-ELS or bisacodyl, prior alimentary tract surgery, or refusal to participate. The research pharmacist used a randomization schedule generated by the website http://www.randomization.com to randomly assign all eligible patients to one of the three bowel preparation regimens. Patients were enrolled in the order they were scheduled for colonoscopy, and the sequence was concealed.

\section{Interventions}

Participants were randomized to each of the three treatment arms. Patients on all arms received PEG-ELS. Participants randomized to the conventional bowel preparation (CBP) arm were instructed to ingest the preparation over 2 to 4 hours the day before the procedure at 1800 hours. Patients receiving split-dose PEG-ELS (SDBP), ingested $2 \mathrm{~L}$ orally at 1800 hours the day before the procedure, and the remaining $2 \mathrm{~L}$ in the early morning at least 3 hours before their scheduled colonoscopy time. Participants in the SDBP-B group received bisacodyl $10 \mathrm{mg}$ orally at 1200 hours the day prior to the procedure, followed by $2 \mathrm{~L}$ of PEG-ELS at 1800 hours the day before the procedure and $2 \mathrm{~L}$ in the early morning at least 3 hours before their scheduled colonoscopy time. Participants in all three arms were placed on a clear liquid diet for the 2 days prior to colonoscopy. Colonoscopies were performed with moderate sedation by one of our nine experienced endoscopists with or without a gastroenterology fellow. All endoscopists were blinded to the preparation regimen and received training and occasional reminders regarding the Boston Bowel Preparation Score (BBPS).

\section{Data collection}

After informed consent and prior to ingesting any bowel preparation, participants were asked to complete a survey regarding gastrointestinal symptoms (dyspepsia, distension, bloating, diarrhea or constipation, nausea or vomiting, abdominal pain) and severity of their diabetes (duration, use of insulin, history of diabetic retinopathy, nephropathy, peripheral paresthesia, and history of coronary artery disease). On the day of the colonoscopy after completing the bowel preparation, patients completed a questionnaire to assess the safety and tolerability of the bowel preparation. The questionnaire had eight items: 1) completion of preparation (less than $50 \%$ vs. more than $50 \%$ ); 2) ease of use (easy or neutral, somewhat easy, or difficult); 3 ) willingness to repeat (yes vs. no) in the future if needed; 4) nausea or vomiting (yes vs. no); 5) abdominal bloating (yes vs. no); 6) abdominal pain (yes vs. no); 7) rectal burning sensation (yes vs. no); and 8) sleep disturbances (yes vs. no). Symptoms or illness requiring treatment were considered severe adverse events.

\section{Efficacy assessment}

To measure efficacy of the bowel preparation, we used the Boston Bowel Preparation Scale (BBPS), a previously validated bowel preparation scoring system based on the summation of the preparation scores from three segments of the colon (right colon, transverse colon, and left colon). The total score is a 9point scale that ranges from 0 to 9 . A segment score of 0 describes an unprepared colon segment with mucosa that are not well seen due to solid stool that cannot be cleared. A segment score of 1 denotes that a portion of the mucosa of the colon segment was seen, but other areas of the colon are not well seen due to staining, residual stool, and/or opaque liquid. A segment score of 2 refers to a minor amount of residual staining, small fragments of stool, and/or opaque liquid, but the mucosa of the colon segment are well visualized. A segment score of 3 reflects that the entire mucosa of the colon segment was well visualized with no residual staining, small fragments of stool, and/or opaque liquid. The quality of bowel preparation was graded at the end of the procedure by an endoscopist blinded to the type of preparation and was based on preparation prior to any washing.

\section{Outcomes}

The primary outcome was adequate bowel preparation quality, defined as BBPS $\geq 6$ with no segmental score $<2$, or subjective rating by the endoscopist as "good" or "excellent." Secondary outcomes included excellent bowel preparation, defined as BBPS $\geq 7$, segmental scores, mean BBPS for ascending, trans- 


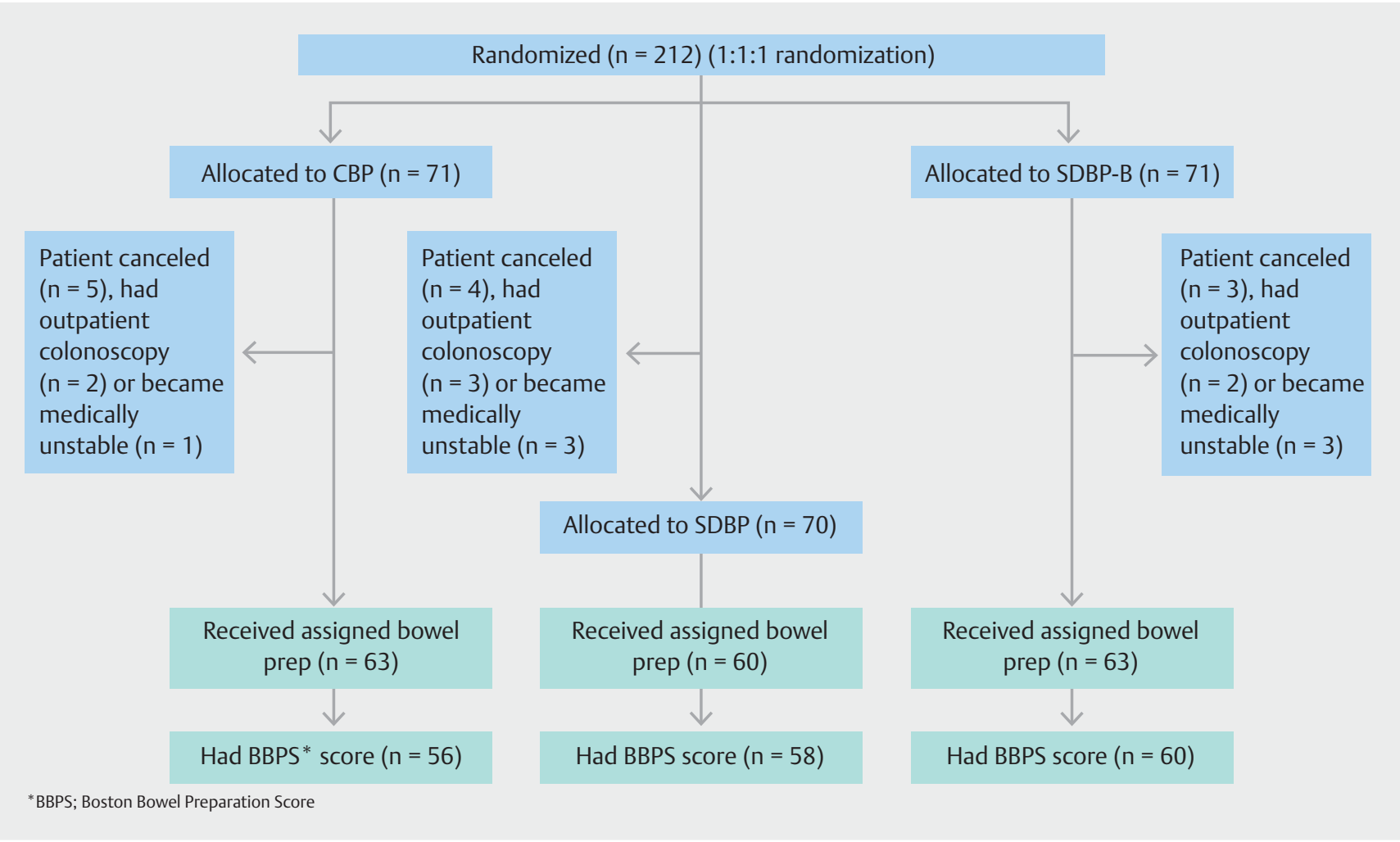

Fig. 1 Flow diagram of patients enrolled.

verse and left colon, percentage of segment score $<2$, tolerability, and safety.

\section{Statistical methods}

The sample size calculation was based on comparison of a preparation quality score between the two groups. We hypothesized that preparation quality of SDBP-B would be superior to that of CBP alone by more than $30 \%$. We assumed that frequency of adequate bowel preparation (defined as BBPS $\geq 6$ with no segmental score $<2$ ) would be $60 \%$ in the CBP arm and $90 \%$ in the SDBP-B arm, based on data from previous studies. A sample size of 62 patients for each group was estimated to give $80 \%$ power at a two-sided alpha of 0.0167 (Bonferroni adjusted for multiple comparisons). We also assumed a drop-out rate of $15 \%$. Hence, the total planned number for enrollment was estimated to be 214 patients. SAS software (SAS Institute, Cary, North Carolina, United States) was used for data analyses. Continuous variables were reported as means $\pm S D$. Categorical variables were reported as percentages. Two-sided $t$-test was used to compare the means of continuous variables in the two groups. Chi-square test was used to compare the categorical variables. A P value $<0.0167$ was considered statistically significant. The intention to treat (ITT) population included all randomized patients who met the inclusion criteria, provided informed consent, and for whom the efficacy of bowel preparation could be assessed.

\section{Results}

More than 600 patients with diabetes were scheduled for outpatient colonoscopy between 2012 and 2016. Of these, 212 patients agreed to participate and were randomized per protocol, with 71 randomized to receive $C B P, 70$ randomized to receive SDBP, and 71 randomized to receive SDBP-B. Of those randomized patients, 26 did not complete the study or receive any of the study-specified bowel preparations. Twelve patients cancelled, seven received their colonoscopies at an outside hospital, and seven became medically unstable ( $\triangleright$ Fig. 1). One hundred and eighty-six participants underwent colonoscopy and were included in the final analysis.

The three groups had similar baseline characteristics. Furthermore, the groups had similar baseline medical comorbidities, duration of diabetes, diabetes-related complications, and use of insulin and other medications, except for tricyclic antidepressants, which were used more often in the group receiving SDBP $(P=0.03$; $>$ Table 1$)$. Cecal intubation rate was similar among the three groups: $63 / 63(100 \%)$ in the CBP arm, 59/60 (98\%) in the SDBP arm, and 63/63 (100\%) in the SDBP-B arm, $P$ $=0.48$. One hundred seventy-four participants had BBPS documentation. The 12 participants who did not have BBPS documentation had objective assessments of the quality of their bowel preparations. 
- Table 1 Baseline characteristics.

\begin{tabular}{|c|c|c|c|c|}
\hline & $\begin{array}{l}\text { CBP } \\
(n=63)\end{array}$ & $\begin{array}{l}\text { SDBP } \\
(n=60)\end{array}$ & $\begin{array}{l}\text { SDBP-B } \\
(n=63)\end{array}$ & $P$ value \\
\hline Age, mean $\pm S D$ & $64.7 \pm 6.4$ & $62.8 \pm 7.2$ & $63.2 \pm 6.7$ & 0.25 \\
\hline Male sex, n (\%) & $61(96.8)$ & $59(98.3)$ & $58(92.1)$ & 0.19 \\
\hline White race, $\mathrm{n}(\%)$ & $41(65.1)$ & $45(75)$ & $51(80.9)$ & 0.14 \\
\hline Indication (FIT positive), n (\%) & $52(82.5)$ & $44(73.3)$ & $48(76.2)$ & 0.46 \\
\hline DM Duration < 5 yr, $n(\%)$ & $23(36.5)$ & $26(43.3)$ & $24(38.1)$ & 0.72 \\
\hline Insulin use, n (\%) & $23(36.5)$ & $21(35)$ & $24(38.1)$ & 0.94 \\
\hline $\mathrm{HgbA} 1 \mathrm{c}$ mean $\pm S D$ & $7.3 \pm 1.4$ & $7.4 \pm 1.8$ & $7.4 \pm 1.6$ & 0.87 \\
\hline Diabetic retinopathy, n (\%) & $7(11.1)$ & $7(11.7)$ & $6(9.5)$ & 0.92 \\
\hline Diabetic nephropathy, n (\%) & $2(3.2)$ & $2(3.3)$ & $3(4.8)$ & 0.87 \\
\hline Diabetic neuropathy, n (\%) & $38(60.3)$ & $39(65)$ & $41(65.1)$ & 0.81 \\
\hline History of CAD, $n(\%)$ & $17(26.9)$ & $23(38.3)$ & $23(36.5)$ & 0.36 \\
\hline Cirrhosis, n (\%) & $1(1.6)$ & $4(6.7)$ & $4(6.4)$ & 0.33 \\
\hline Stroke, n (\%) & $3(4.8)$ & $4(6.7)$ & $6(9.5)$ & 0.57 \\
\hline Dementia, n (\%) & $0(0)$ & $1(1.7)$ & $1(1.6)$ & 0.59 \\
\hline Narcotics use, n (\%) & $21(33.3)$ & $18(30)$ & $24(38.1)$ & 0.63 \\
\hline $\mathrm{CCB}, \mathrm{n}(\%)$ & $19(30.1)$ & $22(36.7)$ & $23(36.5)$ & 0.68 \\
\hline TCA, n (\%) & $3(4.8)$ & $10(16.7)$ & $3(4.8)$ & 0.03 \\
\hline
\end{tabular}

diabetes mellitus; CCB, calcium channel blocker; TCA, tricyclic antidepressant; SD, standard deviation

\section{Primary and secondary outcomes}

Most participants achieved adequate bowel preparation (total BBPS score $\geq 6$, with all segment scores $\geq 2$ : $42 / 63(66.7 \%)$ in the CBP arm, 50/60 (83.3\%) in the SDBP arm, and $47 / 63$ $(74.6 \%)$ in the SDP-B arm; $P=0.10)$. Including the 12 patients who did not have BBPS scores, but had another subjective rating of bowel preparation, showed comparable results $(76.2 \%$ CBP, $85 \%$ SDBP, $77.8 \%$, SDP-B; $P=0.64)$. The mean BBPS was $6.7 \pm 1.5$ for participants on the CBP arm, compared with $7.3 \pm$ 1.6 for patients on the SDBP arm and $7.1 \pm 1.4$ for participants on the SDBP-B arm ( $P=0.26$; $>$ Table 2$)$.

Data for each colon segment were analyzed. More patients in the CBP arm (14/56, 25\%) had BBPS scores <2 in the right side than did patients in the SDBP arm $(5 / 58,10.3 \%)$ and the SDBP-B arm (9/60, 15\%). This difference neared statistical significance $(P=0.02)$. Similarly, the mean right-side segmental BBPS score was significantly lower among patients in the CBP arm $(1.9 \pm 0.6)$ than among patients in the SDBP $(2.3 \pm 0.6)$ or SDBP-B $(2.1 \pm 0.6)$ arms ( $P=0.003$; $>$ Table 2$)$.

\section{Safety and tolerability}

Most participants in all treatment arms completed $>50 \%$ of their assigned bowel preparation. About two-thirds of participants felt that the preparation was either easy or neutral. Incidence of treatment-related side effects was similar between the groups. There was a trend towards more willingness to repeat the procedure (SDBP, $90 \%$; SDBP-B, $92 \%$; CBP, $84 \%$ ) and more loss of sleep (SDBP, 50\%; SDBP-B, 54\%; CBP, 44\%) in the both split-dose groups relative to the CBP group ( $\vee$ Table 3 ).

There were no serious adverse events. One patient in the SDBP group presented the morning of the procedure with new-onset atrial fibrillation, which was felt to be unrelated to the bowel preparation.

\section{Discussion}

In this randomized controlled trial (RCT), adding bisacodyl to SDBP did not improve the quality of bowel preparation in patients with DM. To our knowledge, this is the first RCT to evaluate various bowel preparation in patients with DM.

Gastrointestinal symptoms are more common in patients with DM than in the general population [8]. The most common gastrointestinal complaint is constipation, occurring in up to $60 \%$ of patients [9]. Prolonged whole gut and colonic transient times have been observed in $40 \%$ of patients with diabetes [9]. Gastroparesis is also common [8]. Delayed gastric emptying can be demonstrated in $27 \%$ to $65 \%$ of patients with type I DM, and approximately $30 \%$ of patients with type II DM[10]. Need for colonoscopy is common in patients with DM, given the high prevalence of gastrointestinal symptoms and a possible increased risk of colon cancer $[11,12]$. Previous studies sug- 
- Table2 Quality of bowel preparation.

\begin{tabular}{|c|c|c|c|c|}
\hline & $\begin{array}{l}\text { CBP } \\
(n=63)\end{array}$ & $\begin{array}{l}\text { SDBP } \\
(n=60)\end{array}$ & $\begin{array}{l}\text { SDBP-B } \\
(n=63)\end{array}$ & $P$ value \\
\hline \multicolumn{5}{|l|}{ Aggregate Score } \\
\hline $\mathrm{BBPS} \geq 6, \mathrm{n}(\%)^{1}$ & $42(66.7)$ & $50(83.3)$ & $47(74.6)$ & 0.10 \\
\hline $\mathrm{BBPS} \geq 7, \mathrm{n}(\%)^{1}$ & $31(49.2)$ & $37(61.7)$ & $38(60.3)$ & 0.30 \\
\hline Adequate, n (\%) & $48(76.2)$ & $51(85)$ & $49(77.8)$ & 0.64 \\
\hline Overall BBPS, mean \pm SD & $6.7 \pm 1.5$ & $7.3 \pm 1.6$ & $7.1 \pm 1.4$ & 0.13 \\
\hline \multicolumn{5}{|l|}{ Segment score ${ }^{2}$} \\
\hline Right side BBPS <2, n (\%) & $14 / 56(25)$ & $5 / 58(10.3)$ & $9 / 60(15)$ & 0.02 \\
\hline Transverse BBPS $<2, \mathrm{n}(\%)$ & $4 / 56(7.1)$ & $1 / 58(3.5)$ & $3(5)$ & 0.49 \\
\hline Left side BBPS $<2, \mathrm{n}(\%)$ & $3 / 56(5.4)$ & $3 / 58(5.2)$ & $1 / 60(1.7)$ & 0.76 \\
\hline Right side, mean $\pm S D$ & $1.9 \pm 0.6$ & $2.3 \pm 0.6$ & $2.1 \pm 0.6$ & 0.003 \\
\hline Transverse, mean \pm SD & $2.4 \pm 0.6$ & $2.6 \pm 0.6$ & $2.4 \pm 0.6$ & 0.18 \\
\hline Left side, mean $\pm S D$ & $2.4 \pm 0.6$ & $2.5 \pm 0.6$ & $2.6 \pm 0.5$ & 0.48 \\
\hline \multicolumn{5}{|c|}{$\begin{array}{l}\text { CBP, conventional bowel preparation; SDBP, split-dose bowel preparation; SDBP-B, split-dose bowel preparation plus bisacodyl; BBPS, Boston bowel preparation } \\
\text { score; SD, standard deviation } \\
{ }^{1} \text { Calculation based on all patients, not only to those with available BBPS } \\
{ }^{2} \text { Calculation based on those patients with available BBPS only }\end{array}$} \\
\hline
\end{tabular}

- Table 3 Safety and tolerability.

\begin{tabular}{|c|c|c|c|c|}
\hline & $\begin{array}{l}\text { CBP } \\
\text { n (\%) }\end{array}$ & $\begin{array}{l}\text { SDBP } \\
\text { n (\%) }\end{array}$ & $\begin{array}{l}\text { SDBP-B } \\
\text { n (\%) }\end{array}$ & $P$ value \\
\hline Completed $>50 \%$ & $61(98.9)$ & $56(98.3)$ & $63(100)$ & 0.58 \\
\hline Found to be easy or neutral & $45(72.3)$ & $43(75.4)$ & $50(79.5)$ & 0.67 \\
\hline Nausea/vomiting & $6(9.7)$ & $4(6.9)$ & $6(9.5)$ & 0.85 \\
\hline Bloating & $10(16.1)$ & $10(17.2)$ & $10(15.9)$ & 0.97 \\
\hline Abdominal pain & $2(3.2)$ & $4(6.9)$ & $2(3.2)$ & 0.51 \\
\hline Rectal burning sensation & $6(9.7)$ & $9(15.6)$ & $10(15.9)$ & 0.52 \\
\hline Loss of sleep & $27(43.6)$ & $29(50)$ & $34(53.9)$ & 0.51 \\
\hline Willingness to repeat same bowel preparation & $52(83.9)$ & $52(89.7)$ & $58(92.1)$ & 0.30 \\
\hline
\end{tabular}

gested that the rate of inadequate bowel preparation ranged between $20 \%$ and $38 \%$ in patients with diabetes [13-16].

Few studies have explored ways to improve bowel preparation in this group of patients. Three studies examined alternative strategies to improve quality of bowel preparation in patients with DM using a conventional single-dose PEG-ELS ingested the night before the procedure. One study used $6 \mathrm{~L}$ of PEGELS, and found the rate of inadequate bowel preparation to be around $38 \%$ [13]. In another study, investigators used lubiprostone as an adjunct to a single dose of $4 \mathrm{~L}$ PEG-ELS. An improvement from $24 \%$ good or excellent bowel preparation to $47 \%$ was observed; this difference was not statistically significant [17]. Finally, Hayes et al. explored the option of adding two do- ses of magnesium citrate to the usual single-dose PEG-ELS. A statistically significant improvement in good bowel preparation was reported (54\% vs. $70 \%$ ) [18]. The rate of inadequate bowel preparation was noticeably high in all three studies, and higher than the rate of inadequate bowel preparation observed in the conventional group in the current study (25\%). This study was different in that we required a clear liquid diet for 2 days, and all patients attended an educational class prior to their colonoscopy. These differences could explain the lower rate of inadequate bowel preparation in the conventional arm observed here.

Split-dose PEG-ELS has been shown to be a more effective strategy for improving bowel preparation, and current guide- 
lines recommend its use in all patients [19]. While our study did not demonstrate a difference in adequate bowel preparation in patients receiving SDBP compared with CBP, there was an improvement in the right colon scores. There has been other limited evidence to suggest this regimen improves bowel preparation in patients with DM. One recently published study examined the effect of a multifactorial strategy combining an educational intervention, 4 days of a low-fiber diet, and adjustment of blood glucose-lowering agents on the quality of bowel preparation among patients with DM receiving split-dose bowel preparation. The researchers found that the multifactorial strategy resulted in more adequate bowel preparation compared with the conventional protocol: low-fiber diet for 4 days and clear liquid diet the day before the procedure ( $93 \%$ vs. $80 \%, P=0.014$ ) [15]. Similarly, we found that $82 \%$ of patients in the split-dose groups achieved adequate bowel preparation, which is comparable to the rate of adequate bowel preparation in the AlvarezGonzalez study's standard split-dose treatment arm (80\%). These results clearly demonstrate that inadequate bowel preparation is common in diabetic patients, despite following the current guidelines [19]. The current guidelines recommend a full liquid diet in patients who are at high risk of inadequate coIon preparation, e.g., patients with DM [19]. Concerns about hypoglycemia, which could theoretically lead to less adherence to laxative intake along with the possibility of further impairment of motility function, have been raised. In the study published by Alvarez-Gonzalez, the duration of clear liquid diet was 8 hours in the multifactorial strategy group, compared with 24 hours in the standard care group. In the current study, our patients were asked to follow a clear liquid diet for 2 days before colonoscopy. The duration of clear liquid diet likely plays some role in the quality of bowel preparation in patients with DM. It is possible that less time on clear liquid diet results in better bowel preparation and while this merits further investigation it is unclear how our prolonged diet of clear liquids may have impacted our results

The mechanism of motility dysfunction in patients with DM is unclear. It has been suggested that this dysfunction may be due to autonomic neuropathy of the gastrointestinal tract [20] or to hyperglycemia [21]. Studies also have shown that the colonic smooth muscle can be stimulated in patients with DM [20]. Bisacodyl is a prokinetic with a hydragogue effect, which acts locally in the large bowel by directly enhancing motility and reducing transit time [22]. Our study did not show an improvement in bowel preparation among patients who received bisacodyl and split-dose bowel preparation, which suggests that motility dysfunction is more complex and may not be improved by a stimulant laxative.

Studies have suggested that a split-dose PEG-ELS enhances patients' compliance and tolerability of the preparation by increasing the time required to consume the entire volume of lavage solution. In a meta-analysis of RCTs in adult patients comparing the effect of split-dose PEG-ELS with conventional dose PEG-ELS, split-dose PEG-ELS resulted in reduced nausea, improved bowel preparation, improved patient compliance, and increased willingness to repeat the same preparation [23]. In the current study, we did not observe a statistically significant difference between the split-dose groups and conventional group with regard to tolerability. However, consistent with other trials, a numerical trend towards more willingness to repeat procedure and loss of sleep was observed.

There are some limitations to our study. The study was conducted at a VA medical center in a patient population with the majority of patients being male and white. Thus, these results may not be generalizable to the general population. In addition, it is recommended that the BBPS be documented after the endoscopist has performed the colon washing. In the current study, we required documentation of the bowel preparation score prior to any washing to assess the impact of the bowel preparation rather than the intraprocedural time spent washing. While endoscopists were reminded of this protocol for BPPS documentation, it is possible that end of procedure scoring, which replicates clinical practice, may have impaired scoring accuracy. The high rate of CBP adequate preparation compared to other trials might have limited the power to detect a difference.

\section{Conclusion}

In conclusion, adding bisacodyl to SDBP did not improve the quality of bowel preparation in diabetic patients. Further efforts are needed to optimize bowel preparation in this population.

\section{Acknowledgements}

Grant support was provided by South Central VA Healthcare Network (SCVAHCN).

\section{Competing interests}

None

References

[1] Winawer S], Zauber AG, Ho MN. The National Polyp Study Workgroup. et al. Prevention of colorectal cancer by colonoscopic polypectomy. N Engl J Med 1993; 329: 1977 - 1981

[2] Kim WH, Cho Y], Park JY et al. Factors affecting insertion time and patient discomfort during colonoscopy. Gastrointest Endosc 2000; 52: $600-605$

[3] Ness RM, Manam R, Hoen $\mathrm{H}$ et al. Predictors of inadequate bowel preparation for colonoscopy. Am J Gastroenterol 2001; 96: 1797 1802

[4] Rex DK, Imperiale TF, Latinovich DR et al. Impact of bowel preparation on efficiency and cost of colonoscopy. Am J Gastroenterol 2002; 97: $1696-1700$

[5] Harewood GC, Sharma VK, de Garmo P. Impact of colonoscopy preparation quality on detection of suspected colonic neoplasia. Gastrointest Endosc 2003; 58: 76-79

[6] Fayad NF, Kahi C], Abd El-Jawad KH et al. Association between body mass index and quality of split bowel preparation. Clin Gstroenterolo Hepatol 2013; 11: 1478-1485 
[7] Dik VK, Moons LM, Huyuk M et al. Predicting inadequate bowel preparation for colonoscopy in participants receiving split-dose bowel preparation: development and validation of a prediction score. Gastrointest Endosc 2015; 81: 665-672

[8] Bytzer P, Talley NJ, Leemon M et al. Prevalence of gastrointestinal symptoms associated with diabetes mellitus: a population-based survey of 15,000 adults. Arch Intern Med 2001; 161: 1989-1996

[9] Feldman M, Schiller LR. Disorders of gastrointestinal motility associated with diabetes mellitus. Ann Intern Med 1983; 98: 378-384

[10] Parkman HP, Yates K, Hasler WL et al. Similarities and differences between diabetic and idiopathic gastroparesis. Clin Gastroenterol Hepatol 2011; 9: 1056 - 1064; quiz e133-134

[11] La VecchiaC, Negri E, Decarli A et al. Diabetes mellitus and colorectal cancer risk. Cancer Epidemiol Biomarkers Prev 1997; 6: 1007-1010

[12] Will JC, Galuska DA, Vinicor F et al. Colorectal cancer: another complication of diabetes mellitus? Am J Epidemiol 1998; 147: 816-825

[13] Taylor C, Schubert ML. Decreased efficacy of polyethylene glycol lavage solution (golytely) in the preparation of diabetic patients for outpatient colonoscopy: a prospective and blinded study. Am J Gastroenterol 2001; 96: 710 - 714

[14] Ozturk NA, Gokturk HS, Demir M et al. Efficacy and safety of sodium phosphate for colon cleansing in type 2 diabetes mellitus. South Med J 2010; 103: 1097-1102

[15] Alvarez-Gonzalez MA, Flores-Le Roux JA, Seoane A et al. Efficacy of a multifactorial strategy for bowel preparation in diabetic patients undergoing colonoscopy: a randomized trial. Endoscopy 2016; 48: $1003-1009$
[16] Madhoun MF, Bitar H, Bhatti O et al. Diabetics on narcotics are less likely to achieve excellent bowel preparation than are patients with either condition. Dig Dis Sci 2017; 62: 723 - 729

[17] Grigg E, Schubert MC, Hall J et al. Lubiprostone used with polyethylene glycol in diabetic patients enhances colonoscopy preparation quality. World J Gstrointest Endosc 2010; 2: 263-267

[18] Hayes A, Buffum M, Hughes J. Diabetic colon preparation comparison study. Gastroenterol Nurs 2011; 34: 377 - 82

[19] Johnson DA, Barkun AN, Cohen LB et al. Optimizing adequacy of bowel cleansing for colonoscopy: recommendations from the US Multi-Society Task Force on Colorectal Cancer. Am J Gastroenterol 2014; 109: 1528 - 1545

[20] Battle WM, Snape W] Jr, Alavi A et al. Colonic dysfunction in diabetes mellitus. Gastroenterology 1980; 79: 1217-1221

[21] Sims MA, Hasler WL, Chey WD et al. Hyperglycemia inhibits mechanoreceptor-mediated gastrocolonic responses and colonic peristaltic reflexes in healthy humans. Gastroenterology 1995; 108: 350-359

[22] Herve S, Savoye G, Behbahani A et al. Results of 24-h manometric recording of colonic motor activity with endoluminal instillation of bisacodyl in patients with severe chronic slow transit constipation. Neurogastroenterol Motil 2004; 16: $397-402$

[23] Kilgore TW, Abdinoor AA, Szary NM et al. Bowel preparation with split-dose polyethylene glycol before colonoscopy: a meta-analysis of randomized controlled trials. Gastrointest Endosc 2011; 73: 1240 1245 